\title{
A STRUCTURE THEOREM FOR THE COMMUTANT OF A CLASS OF CYCLIC SUBNORMAL OPERATORS
}

\author{
MARC RAPHAEL
}

\begin{abstract}
An $m$-measure is defined to be a measure $\mu$ such that the analytic bounded point evaluations of $P^{2}(\mu)$ is the open unit disk $\mathbf{D}$ in the complex plane, and the weak* closure of the analytic polynomials in $L^{\infty}(\mu)$ is the set of bounded analytic functions on D. A complete characterization of $P^{2}(\mu) \cap L^{\infty}(\mu)$, the commutant of the cyclic subnormal operator of multiplication by $z$ on $P^{2}(\mu)$, is then obtained.
\end{abstract}

In this paper a complete characterization is given of the commutant of a class of cyclic subnormal operators closely related to the unilateral shift.

An operator $S$ on a Hilbert space $\mathscr{H}$ is subnormal if there is a Hilbert space $\mathscr{K}$ containing $\mathscr{H}$ and a normal operator $N$ on $\mathscr{K}$ such that $N(\mathscr{H}) \subseteq \mathscr{H}$ and $S=N \mid \mathscr{H}$ (the restriction of $N$ to $\mathscr{H}$ ). The weak* topology on $B(\mathscr{H})$ is the topology which $B(\mathscr{H})$ has as the Banach space dual of the trace class operators [4].

A measure $\mu$ is always a compactly supported, positive regular Borel measure on the complex plane, $\mathbf{C}$. If $S$ is a cyclic subnormal operator, then there exists a measure $\mu$ such that $S$ is unitarily equivalent to $S_{\mu}$, the operator of multiplication by $z$ on $P^{2}(\mu)=$ the closure of the analytic polynomials in $L^{2}(\mu)$ [4]. Yoshino's Theorem [4] states that the map from $P^{2}(\mu) \cap L^{\infty}(\mu)$ onto $\left\{S_{\mu}\right\}^{\prime}=$ the commutant of $S_{\mu}$, given by $\phi \mapsto \phi\left(S_{\mu}\right)=$ multiplication by $\phi$, is an isometric isomorphism and a weak* homeomorphism. For functions $f, g$ in $L^{2}(\mu),\langle f, g\rangle=\int f \bar{g} d \mu,\|f\|_{2}=$ $(\langle f, f\rangle)^{1 / 2}$, and $\|f\|_{\mu}$ denotes the $\mu$-essential supremum of $f$. Let $m$ denote normalized arc length measure on $\partial \mathbf{D}$, the boundary of the open unit disk. Thus $S_{m}$ is the unilateral shift. A class of measures with many of the properties of $m$ will be defined after some notation is set.

If $\mu$ is a measure, then $B(\mu)$, the set of bounded point evaluations of $P^{2}(\mu)$, consists of those $\lambda$ in $\mathbf{C}$ for which the linear functional $p \mapsto p(\lambda)$ has a bounded extension from the polynomials to $P^{2}(\mu)$. Equivalently, $\lambda \in B(\mu)$ if and only if there exists $k_{\lambda}$ in $P^{2}(\mu)$ such that $p(\lambda)=\left\langle p, k_{\lambda}\right\rangle$ for all polynomials $p$. $B_{a}(\mu)$, the set of analytic bounded point evaluations of $P^{2}(\mu)$, is the largest open subset of $B(\mu)$ such that the function

$$
\tilde{f}(\lambda)=\left\langle f, k_{\lambda}\right\rangle
$$

is analytic in $B_{a}(\mu)$ for every $f$ in $P^{2}(\mu)$.

Received by the editors February 25, 1985.

1980 Mathematics Subject Classification. Primary 47B20; Secondary, 47C05. 
In view of (1), every $f$ in $P^{2}(\mu)$ can be given a pointwise definition on $B_{a}(\mu)$; namely, $f(\lambda)=\tilde{f}(\lambda)$ for $\lambda$ in $B_{a}(\mu)$. Since the pointwise definition of $f$ agrees $\mu$-a.e. with any Borel function that represents the equivalence class of $f$ in $P^{2}(\mu)$, it will be agreed upon once and for all that each $f$ in $P^{2}(\mu)$ is defined pointwise on $B_{a}(\mu)$ via (1). The $\sim$ notation will be dropped in all instances.

The proof of the following proposition is left to the reader.

Proposition 2. Let $\mu$ be a measure and $\lambda \in B_{a}(\mu)$. If $f \in P^{2}(\mu)$ and $g \in P^{2}(\mu)$ $\cap L^{\infty}(\mu)$, then $f g \in P^{2}(\mu)$ and $(f g)(\lambda)=f(\lambda) g(\lambda)$.

An $m$-measure is defined as a measure $\mu$ with the following two properties:

(a) $B_{a}(\mu)=\mathbf{D}$;

(b) $P^{\infty}(\mu)$, the weak* closure of the analytic polynomials in $L^{\infty}(\mu)$, has no $L^{\infty}$-summand, and the interior of the Sarason hull of $\mu$ is $\mathbf{D}$.

The terminology of condition (b) is taken from [4]. Condition (b) is equivalent to the identity mapping on the polynomials extending to an isometric isomorphism that is a weak* homeomorphism from $P^{\infty}(\mu)$ onto $P^{\infty}(m)$. In particular $\mu$ is supported on $\mathbf{D}$ and $\mu \mid \partial \mathbf{D}<<m$.

Condition (b) will be abbreviated simply to " $P^{\infty}(\mu)=H^{\infty}$ ". It is not a substantial restriction on $\mu$ as is indicated by Theorem 4.11 of [5]. On the other hand, if $P^{2}(\mu) \neq L^{2}(\mu)$, then it is unknown whether $B(\mu)$ is nonempty.

EXAMPLE 3. Some examples of $m$-measures are now given.

(i) Of course $m$ is an $m$-measure.

(ii) Area measure on $\mathbf{D}$ is an $m$-measure.

(iii) If $\mu$ is a measure supported on $\mathbf{D}^{-}$such that $\mu \mid \partial \mathbf{D}<<m$ and $d(\mu \mid \partial \mathbf{D}) / d m$ is log-integrable with respect to $m$, then $\mu$ is an $m$-measure. This follows by combining Theorem 4.5 of [2] with Corollary 5 of [8].

(iv) Let $\left\{a_{n}\right\}$ be a sequence in $\mathbf{D}$ with $\partial \mathbf{D} \subseteq\left\{a_{n}\right\}^{-}$. If almost every point of $\partial \mathbf{D}$ can be approached nontangentially by a subsequence of $\left\{a_{n}\right\}$, then $\mu=\Sigma 2^{-n} \delta_{a_{n}}$ is an $m$-measure [1]. Here, $\delta_{a_{n}}$ is the unit point mass measure at $a_{n}$.

(v) If $\nu$ is a probability measure on $[0,1]$ with 1 in the support of $\nu$, then $d \mu\left(r e^{i \theta}\right)=d \nu(r) d m\left(e^{i \theta}\right)$ is an $m$-measure and $S_{\mu}$ is the canonical model for a subnormal weighted shift operator of norm $1[4]$.

(vi) Let $G=\mathbf{D} \backslash\{z \in \mathbf{C}:|z-1 / 2| \leqslant 1 / 2\}$. Using a technique from [6], one can construct a measure $\mu$, equivalent to area measure on $G$, such that $P^{\infty}(\mu)=H^{\infty}$ and $B_{a}(\mu)=G$. Thus $\mu$ is not an $m$-measure since $B_{a}(\mu) \neq \mathbf{D}$.

The main result of this paper is the following.

THEOREM 4. If $\mu$ is an m-measure, then there is a unique $\mu$-measurable subset $\Delta$ of dD such that:

(a) $P^{2}(\mu) \cap L^{\infty}(\mu)=P^{\infty}(\mu \mid \mathbf{C} \backslash \Delta) \oplus L^{\infty}(\mu \mid \Delta)$;

(b) $\mu \mid \mathbf{C} \backslash \Delta$ is an m-measure;

(c) $P^{2}(\mu \mid \mathbf{C} \backslash \Delta) \cap L^{\infty}(\mu \mid \mathbf{C} \backslash \Delta)=P^{\infty}(\mu \mid \mathbf{C} \backslash \Delta)=H^{\infty}$.

Theorem 4 will be established with the aid of several lemmas. 
Lemma 5. If $\mu$ is an m-measure and $f \in P^{2}(\mu) \cap L^{\infty}(\mu)$, then there is a $g$ in $P^{\infty}(\mu)$ such that $(f-g) \mid \mathbf{D}=0$.

Proof. It can be assumed that $\|f\|_{\mu}<1$. For $\lambda$ in $\mathbf{D}$,

$$
|f(\lambda)|^{n}=\left|\left\langle f, k_{\lambda}\right\rangle\right|^{n}=\left|\left\langle f^{n}, k_{\lambda}\right\rangle\right| \leqslant\left\|f^{n}\right\|_{2}\left\|k_{\lambda}\right\|_{2} \leqslant\left\|k_{\lambda}\right\|_{2} .
$$

Letting $n \rightarrow \infty$ shows that $|f(\lambda)| \leqslant 1$. Since $B_{a}(\mu)=\mathbf{D}, f$ is analytic and bounded on D. Since $P^{\infty}(\mu)=H^{\infty}$, there exists $g$ in $P^{\infty}(\mu)$ such that $(f-g) \mid \mathbf{D}=0$.

LEMMA 6. If $\mu$ is a measure such that $S_{\mu}$ is quasisimilar to $S_{m}$, then $P^{2}(\mu) \cap L^{\infty}(\mu)$ $=P^{\infty}(\mu)=H^{\infty}$.

Proof. According to Theorem 4.5 of [2], a necessary and sufficient condition for $S_{\mu}$ and $S_{m}$ to be quasisimilar is that $\mu$ be a measure as in Example 3(iii). Therefore $P^{\infty}(\mu)=H^{\infty}$ and if $f \in P^{2}(\mu) \cap L^{\infty}(\mu)$, then there exists, by the previous lemma, a $g$ in $P^{\infty}(\mu)$ such that $(f-g) \mid \mathbf{D}=0$. Let $h=f-g$ and choose a sequence of polynomials $\left\{p_{n}\right\}$ that converges to $h$ in $L^{2}(\mu)$. Choose $\phi$ in $H^{2}$ (the Hardy space of square integrable functions) such that $|\phi|^{2}=d \mu_{0} \mid d m$ where $\mu_{0}=\mu \mid \partial \mathbf{D}$. Then

$$
0=\lim _{n \rightarrow \infty} \int_{\partial \mathbf{D}}\left|p_{n}-h\right|^{2} d \mu=\lim _{n \rightarrow \infty} \int\left|p_{n} \phi-h \phi\right|^{2} d m .
$$

It follows that $h \phi \in H^{2}$; so if $P_{\lambda}$ is the Poisson kernel at $\lambda$, then

$$
(h \phi)(\lambda)=\lim _{n \rightarrow \infty} \int P_{\lambda}\left(p_{n} \phi\right) d m=\lim _{n \rightarrow \infty}\left(p_{n} \phi\right)(\lambda)=0 .
$$

Thus $h \phi=0$ as an element of $H^{2}$. Since $\log |\phi| \in L^{1}(m)$, it must be that $h=0$ $m$-a.e.; hence $P^{2}(\mu) \cap L^{\infty}(\mu)=P^{\infty}(\mu)$.

LEMMA 7. Let $\mu$ be an m-measure and $g \in P^{2}(\mu) \cap L^{\infty}(\mu)$. If $g \mid \mathbf{D}=0$ and $E=\{z \in \partial \mathbf{D}: g(z) \neq 0\}$, then $\chi_{E} \in P^{2}(\mu) \cap L^{\infty}(\mu)$.

Proof. If $S_{\mu}$ is quasisimilar to $S_{m}$, then Lemma 6 implies that $P^{2}(\mu) \cap L^{\infty}(\mu)=$ $H^{\infty}$. In this case, $\chi_{E}=0$, so there is nothing to prove.

Now suppose $S_{\mu}$ is not quasisimilar to $S_{m}$. Then $\log \left(d \mu_{0} / d m\right) \notin L^{1}(m)$ where $\mu_{0}=\mu \mid \partial \mathbf{D}$ (Theorem 4.5, [2]). Hence Szegö's Theorem implies that $P^{2}\left(\mu_{0}\right)=L^{2}\left(\mu_{0}\right)$. For $k>0$ let $E_{k}=\left\{z \in \partial \mathbf{D}:|g(z)|>k^{-1}\right\}$, and let $\left\{p_{n}\right\}$ be a sequence of polynomials such that

$$
0=\lim _{n \rightarrow \infty} \int\left|p_{n}-g^{-1} \chi_{E_{k}}\right|^{2} d \mu_{0}=\lim _{n \rightarrow \infty} \int\left|p_{n} g-\chi_{E_{k}}\right|^{2} d \mu .
$$

Thus $\chi_{E_{k}} \in P^{2}(\mu)$. Since $\chi_{E_{k}} \rightarrow \chi_{E}$ weak* in $L^{\infty}(\mu), \chi_{E} \in P^{2}(\mu) \cap L^{\infty}(\mu)$.

Proof of Theorem 4. If $P^{2}(\mu) \cap L^{\infty}(\mu)=P^{\infty}(\mu)$, then it is easy to see that the theorem holds, and $\Delta$ is the empty set.

Now suppose $P^{2}(\mu) \cap L^{\infty}(\mu) \neq P^{\infty}(\mu)$. By Lemma 6 and Theorem 4.5 of [2], $\log \left(d \mu_{0} \mid d m\right) \notin L^{1}(m)$ where $\mu_{0}=\mu \mid \partial \mathbf{D}$. Szegö's Theorem now shows that

$$
\mu(\partial \mathbf{D})<\mu\left(\mathbf{D}^{-}\right) \text {. }
$$

A standard argument shows that $W=\left\{F \subseteq \partial \mathbf{D}: \chi_{F} \in P^{2}(\mu) \cap L^{\infty}(\mu)\right\}$ contains a unique maximal element, $\Delta$. Since $P^{2}(\mu) \cap L^{\infty}(\mu) \neq P^{\infty}(\mu)$, Lemmas 5 and 7 imply $\mu(\Delta)>0$. 
It is now shown that $P^{2}(\mu) \cap L^{\infty}(\mu)=P^{\infty}(\mu \mid \mathbf{C} \backslash \Delta) \oplus L^{\infty}(\mu \mid \Delta)$. If $f \in L^{\infty}(\mu \mid \Delta)$ let $\left\{p_{n}\right\}$ be a sequence of polynomials such that $p_{n} \rightarrow f$ in $L^{2}\left(\mu_{0}\right)=P^{2}\left(\mu_{0}\right)$. Since $p_{n} \chi_{\Delta} \rightarrow f$ in $L^{2}(\mu), f \in P^{2}(\mu) \cap L^{\infty}(\mu)$. Now suppose that $f \in P^{\infty}(\mu \mid \mathbf{C} \backslash \Delta)$. Let $\left\{p_{\alpha}\right\}$ be a net of polynomials that converges to $f$ weak* in $L^{\infty}(\mu \mid \mathbf{C} \backslash \Delta)$. Since the net $\left\{p_{\alpha}\right\}$ converges to $f$ weak $^{*}$ in $L^{2}(\mu \mid \mathbf{C} \backslash \Delta)$, a sequence consisting of convex combinations of elements from the set $\left\{\left(1-\chi_{\Delta}\right) p_{\alpha}\right\}$ converges to $f$ in $L^{2}(\mu)$. This shows that $P^{\infty}(\mu \mid \mathbf{C} \backslash \Delta) \oplus L^{\infty}(\mu \mid \Delta) \subseteq P^{2}(\mu) \cap L^{\infty}(\mu)$.

To show the reverse inclusion, let $f \in P^{2}(\mu) \cap L^{\infty}(\mu)$ and select $g$ in $P^{\infty}(\mu)$ such that $(f-g) \mid \mathbf{D}=0$ (Lemma 5). If $E=\{z \in \partial \mathbf{D}:(f-g)(z) \neq 0\}$, then according to Lemma 7 ,

$$
\chi_{E} \in P^{2}(\mu) \cap L^{\infty}(\mu) .
$$

By the maximality of $\Delta, E \subseteq \Delta$; so $\left(1-\chi_{\Delta}\right)(f-g)=0$. Therefore $\left(1-\chi_{\Delta}\right) f=$ $\left(1-\chi_{\Delta}\right) g \in P^{\infty}(\mu \mid \mathbf{C} \backslash \Delta)$. Since $f=\left(1-\chi_{\Delta}\right) f+\chi_{\Delta} f$, (a) of Theorem 4 holds.

Now, for notational convenience, let $\nu=\mu\left|\mathbf{C} \backslash \Delta, \nu^{\prime}=\mu\right| \Delta$, and $\sigma_{\text {ap }}(S)$ be the approximate point spectrum of $S$. Since $S_{\mu}=S_{\nu} \oplus S_{\nu^{\prime}}$ and $S_{\nu^{\prime}}$ is normal, it follows that

$$
\sigma\left(S_{\nu}\right) \backslash \sigma_{\text {ap }}\left(S_{\nu}\right) \supseteq \sigma\left(S_{\nu}\right) \backslash \sigma_{\text {ap }}\left(S_{\mu}\right)=\sigma\left(S_{\mu}\right) \backslash \sigma_{\text {ap }}\left(S_{\mu}\right) \text {. }
$$

By Theorem 1.1 of [9], $B_{a}(\nu) \supseteq B_{a}(\mu)=\mathbf{D}$; hence $B_{a}(\nu)=\mathbf{D}$. Since, in general, $B_{a}(\nu)$ is contained in the interior of the Sarason hull of $\nu$, it will follow that $\nu$ is an $m$-measure if $P^{\infty}(\nu)$ has no $L^{\infty}$-summand. Suppose $L^{\infty}(\nu \mid \Sigma)$ is an $L^{\infty}$-summand of $P^{\infty}(\nu)\left(\right.$ viz. $\left.P^{\infty}(\nu)=P^{\infty}(\nu \mid \mathbf{C} \backslash \Sigma) \oplus L^{\infty}(\nu \mid \Sigma)\right)$. Since $B_{a}(\nu)=\mathbf{D}, \Sigma \subseteq \partial \mathbf{D} \backslash \Delta$. This contradicts the maximality of $\Delta$; thus (b) of Theorem 4 holds.

To obtain (c), apply what has already been proved to the $m$-measure $\mu \mid \mathbf{C} \backslash \Delta$ and use the maximality of $\Delta$.

To prove uniqueness of $\Delta$ suppose that $\Delta_{1}$ is any subset of $\partial \mathbf{D}$ that satisfies the conditions of the theorem. Since $\chi_{\Delta_{1}} \in P^{2}(\mu) \cap L^{\infty}(\mu)$ and $\Delta$ is the unique maximal element of $W, \Delta_{1} \subseteq \Delta$. By (c), $\chi_{\Delta \backslash \Delta_{1}} \in P^{2}\left(\mu \mid \mathbf{C} \backslash \Delta_{1}\right) \oplus L^{\infty}\left(\mu \mid \Delta_{1}\right)=H^{\infty}$. If $\chi_{\Delta \backslash \Delta_{1}} \neq 0$, then $\mu\left(\Delta \backslash \Delta_{1}\right)=\mu(\partial D)$. It then follows that $\chi_{\Delta \backslash \Delta_{1}}(z)=1$ for every $z$ in D. This contradicts (8) and establishes the theorem.

This paper concludes with four easy consequences of Theorem 4 . The following notation will be useful.

Notation 9. (i) Assume $\mu$ is an $m$-measure and $\phi \in P^{2}(\mu) \cap L^{\infty}(\mu)$.

(ii) $N_{\mu}$ is the normal operator of multiplication by $z$ on $L^{2}(\mu)$.

(iii) $\phi\left(S_{\mu}\right)$ and $\phi\left(N_{\mu}\right)$ are the operators of multiplication by $\phi$ on $P^{2}(\mu)$ and $L^{2}(\mu)$, respectively.

(iv) If $\mu$ is an $m$-measure and $\Delta$ is as in Theorem 4 , then $\mu_{1}=\mu \mid \mathbf{C} \backslash \Delta$ and $\mu_{2}=\mu \mid \Delta$. The decomposition of $\phi$ with respect to $P^{2}(\mu) \cap L^{\infty}(\mu)=P^{\infty}\left(\mu_{1}\right) \oplus$ $L^{\infty}\left(\mu_{2}\right)$ is $\phi=\phi_{1} \oplus \phi_{2}$.

COROLlary 10. Suppose that $\mu$ and $\nu$ are m-measures. If $S_{\mu}$ and $S_{\nu}$ are quasisimilar, then $\left\{S_{\mu}\right\}^{\prime}$ is isometrically isomorphic and weak ${ }^{*}$ homeomorphic to $\left\{S_{\mu}\right\}^{\prime}$ via a map that takes $S_{\mu}$ to $S_{\nu}$. 
Proof. Let $\Delta, \Sigma \subseteq \partial \mathbf{D}$ be the sets given by Theorem 4 in the decomposition of $P^{2}(\mu) \cap L^{\infty}(\mu)$ and $P^{2}(\nu) \cap L^{\infty}(\nu)$, respectively. The decomposition of $S_{\mu}$ and $S_{\nu}$ into their pure and normal parts is

$$
S_{\mu}=S_{\mu_{1}} \oplus N_{\mu_{2}} \quad \text { and } \quad S_{\nu}=S_{\nu_{1}} \oplus N_{\nu_{2}} .
$$

Since $N_{\mu_{2}}$ and $N_{\nu_{2}}$ are unitarily equivalent [3], $L^{\infty}\left(\mu_{2}\right)=L^{\infty}\left(\nu_{2}\right)$. The map is now obvious since $P^{\infty}\left(\mu_{1}\right)=H^{\infty}=P^{\infty}\left(\nu_{1}\right)$.

Corollary 11. Let $\mu$ be an m-measure and $\phi=\phi_{1} \oplus \phi_{2}$ be in $P^{2}(\mu) \cap L^{\infty}(\mu)$. Then:

(a) the minimal normal extension of $\phi\left(S_{\mu}\right)$ is $\phi\left(N_{\mu}\right)$ if and only if $\phi_{1}$ is not constant;

(b) if $\phi_{1}$ is constant, then $\phi\left(S_{\mu}\right)$ is normal;

(c) $\sigma\left(\phi\left(S_{\mu}\right)\right)=\phi(\mathbf{D})^{-} \cup\left\{\mu_{2}\right.$-essential range of $\left.\phi_{2}\right\}$.

Proof. Corollary 11 is a direct result of Corollary 3.2 and Theorem 4.2 of Chapter VIII of [4].

The proof of the next corollary is left to the reader.

Corollary 12. Let $\mu$ be an m-measure. A subspace $\mathscr{M}$ of $P^{2}(\mu)$ is a hyperinvariant subspace for $S_{\mu}$ if and only if $\mathscr{M}=\mathscr{M}_{1} \oplus \mathscr{M}_{2}$ where $\mathscr{M}_{1}$ is an invariant subspace for $S_{\mu_{1}}$ and $M_{2}$ is a reducing subspace for $N_{\mu_{2}}$.

If $\mu$ is any measure, Theorems 2 and 3 of [7] show that $S_{\mu}$ has an invariant subspace that is not hyperinvariant if and only if $P^{2}(\mu) \cap L^{\infty}(\mu) \neq P^{\infty}(\mu)$. Thus Theorem 4 yields the following result.

Corollary 13. Let $\mu$ be an m-measure and $\Delta$ be as in Theorem 4. Then $S_{\mu}$ has an invariant subspace that is not hyperinvariant if and only if $\Delta$ is nonempty.

ACKNOWLEDGMENT. The results in this paper are part of the author's $\mathrm{Ph}$. D. thesis written at Indiana University under the direction of Professor John B. Conway. The author wishes to express his appreciation for the encouragement and guidance of Professor John B. Conway.

\section{BIBLIOGRAPHY}

1. L. Brown, A. L. Shields and K. Zellar, On absolutely convergent sums, Trans. Amer. Math. Soc. 96 (1960), 162-183.

2. W. S. Clary, Quasi-similarity and subnormal operators, Ph. D. thesis, University of Michigan, 1973.

3. J. B. Conway, On quasisimilarity for subnormal operators, Illinois J. Math. 24 (1980), 689-702.

4. __ Subnormal operators, Pitman, Marshfield, Mass., 1981.

5. J. B. Conway and R. F. Olin, A functional calculus for subnormal operators. II, Mem. Amer. Math. Soc., Vol. 10, No. 184 (1977).

6. W. W. Hastings, A construction of Hilbert spaces of analytic functions, Proc. Amer. Math. Soc. 74 (1979), 295-298.

7. R. F. Olin and J. E. Thomson, Algebras of subnormal operators, J. Funct. Anal. 37 (1980), 271-301

8. M. Raphael, Quasisimilarity and essential spectra for subnormal operators, Indiana Univ. Math. J. 31 (1982), 243-246.

9. T. T. Trent, $H^{2}(\mu)$ spaces and bounded point evaluations, Pacific J. Math. 80 (1979), 279-292.

Department of Mathematics, University of Missouri, Rolla, Missouri 65401 